\title{
Design and Implementation of a MASH2-0 Electromechanical Sigma-Delta Modulator for Capacitive MEMS Sensors Using Dual Quantization Method
}

\author{
Bader Almutairi, Ali Alshehri, and Michael Kraft
}

\begin{abstract}
In this paper, a new control structure based on the dual quantization technique is presented for an electromechanical sigma-delta modulator (EM- $\Sigma \Delta M)$ applied to a microelectromechanical system (MEMS) accelerometer. The modulator adopts a 2-0 multistage noise shaping structure (MASH2-0), taking the advantage of the intrinsic linearity of single-bit quantization and the reduced quantization error of multibit quantization in a single modulator. The sensor system is studied by the system-level modeling and the hardware implementation based on the field-programmable gate array technology. The study shows that, MASH2-0 shares the benefits of a MASH2-2 architecture of having an inherent stability, a high overload input level, and a high dynamic range compared with single-loop EM- $\Sigma \Delta M$. However, the MASH2-0 architecture benefits from a considerably simpler implementation, while achieving a higher dynamic range and a higher signal-to-noise ratio compared with a MASH2-2 and a fourth-order single-loop $\Sigma \Delta M$ architecture. A capacitive MEMS accelerometer was designed and employed with this control system. Within a bandwidth of $1 \mathrm{kHz}$, the sensor achieved a noise floor level of $-130 \mathrm{~dB}$ and a bias instability as low as $20 \mu \mathrm{g}$ at an integration time of $40 \mathrm{~s}$. Simulation estimated a full scale of $\pm 20 \mathrm{~g}$ acceleration. The investigation confirms the concept of the MASH2-0 structure and shows its potential as a closed-loop interface for high-performance capacitive MEMS accelerometers.

[2015-0071]
\end{abstract}

Index Terms-Sigma-delta modulator, multi stage noise shaping, MASH, MEMS accelerometer, digital filters.

\section{INTRODUCTION}

$\mathbf{T}$ HE CONCEPT of the electromechanical sigma delta modulator (EM- $\Sigma \Delta \mathrm{M})$ was initially introduced by Henrion et al. [1] with a second order EM- $\Sigma \Delta \mathrm{M}$ in which the micromechanical accelerometer sensing element solely provided the loop dynamics. It was obvious that the quantization noise was a key concern in such a second order control architecture. Therefore, recent research focused on

Manuscript received March 9, 2015; revised May 19, 2015; accepted May 29, 2015. Subject Editor R. T. Howe.

B. Almutairi and A. Alshehri are with the Space Research Institute, King Abdulaziz City for Science and Technology, Riyadh, Saudi Arabia (e-mail: bmutairi@kacst.edu.sa; ashehri@kacst.edu.sa).

M. Kraft is with the Montefiore Institute, University of Liege, Wallonia, 4000 Liege, Belgium (e-mail: m.kraft@ulg.ac.be).

Color versions of one or more of the figures in this paper are available online at http://ieeexplore.iee.org.

Digital Object Identifier 10.1109/JMEMS.2015.2443077 the ability of high-order EM- $\Sigma \Delta$ Ms to minimize quantization noise and improve the linearity, dynamic range, and bandwidth of capacitive micro-electro-mechanical systems (MEMS) sensors while providing digital output in the form of a pulse density modulated signal. High-order EM- $\Sigma \Delta$ Ms with single loop architecture have successfully been applied to capacitive MEMS accelerometers [2]-[4]. However, increasing the order of these modulators in single loop architecture comes with stability constraints, and can only operate with a lower maximum acceleration input level compared to second-order electromechanical $\Sigma \Delta \mathrm{Ms}$ [5]-[7]. Otherwise, the system will become overloaded and unstable. On the other hand, a high order EM- $\Sigma \Delta \mathrm{M}$ can be achieved by means of multi-stage noise shaping (MASH). The potential advantages of an EM- $\Sigma \Delta$ M MASH compared to single-loop, high-order $\Sigma \Delta$ Ms applied to inertial MEMS sensors are its inherent stability and high overload input level due to the use of lower order $\Sigma \Delta \mathrm{Ms}$ in its individual stages. Furthermore, MASH combines the advantages of high dynamic range and high noise shaping performance because of its overall high-order $\Sigma \Delta \mathrm{M}$ architecture.

The MASH concept was first introduced for electronic analogue to digital convertors (ADC) and typically employs cascaded $\Sigma \Delta \mathrm{Ms}$ of first- and second-order stages along with digital filters [8]-[10]. The MASH structure attains the performance of the high-order, single-loop $\Sigma \Delta \mathrm{M}$ while overcoming the latter's potential instability. However, the MASH structure requires precise digital parameters that match with the analogue components in order to cancel the quantization noise [11], [12]. The Sturdy MASH (S-MASH) is another $\Sigma \Delta \mathrm{M}$ structure that retains MASH performance and does not require digital filters. Thus, the necessity for analogue components and digital filter matching is eliminated [13], [14]. However, the S-MASH requires access to the second integrator of the first stage, thus the implementation of this structure is not applicable for the electromechanical $\Sigma \Delta \mathrm{M}$, as the first stage has an inaccessible node within the micro-fabricated sensing element.

A MASH2-2 EM- $\Sigma \Delta \mathrm{M}$ was successfully applied to a MEMS accelerometer [15], [16], which included a second-order electromechanical $\Sigma \Delta \mathrm{M}$ loop cascaded with a purely electronic second-order $\Sigma \Delta \mathrm{M}$. The quantization noise 


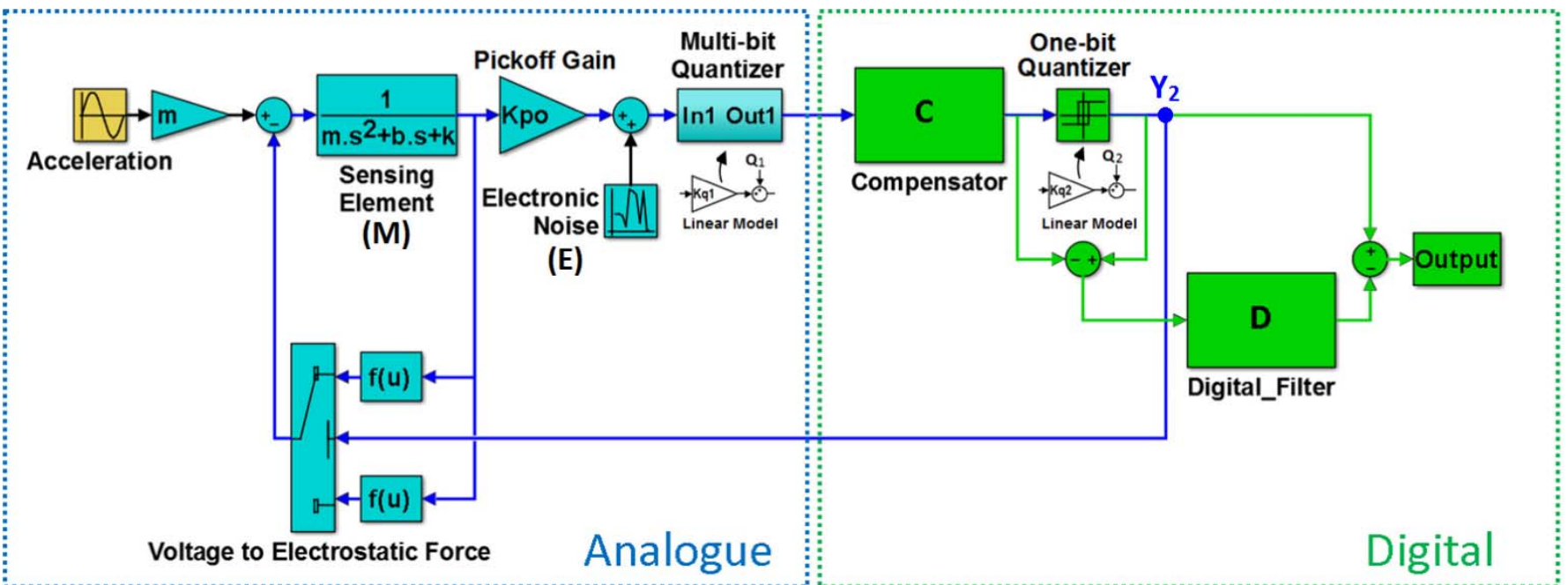

Fig. 1. Simulink model of the MASH2-0 electromechanical $\Sigma \Delta \mathrm{M}$. The analogue part is comprised of a sensing element, a pickoff circuit, a multi-bit quantizer, and an electrostatic feedback unit. The digital part is comprised of a compensator unit, a single-bit quantizer, quantization error extraction, and a digital filter.

from the first loop is digitized by the second loop and then cancelled by digital filters, while the quantization noise from the second loop is shaped by the second loop filter and a digital filter, which together provide fourth order noise shaping. Due to the fact that the MASH structure cascades several $\Sigma \Delta$-modulator stages to achieve high performance, the complexity of the required 1digital filters increases as the number of stages and/or the order of the individual stages increases, which makes digital filter matching hard to achieve.

The dual quantization technique represents another approach to the design of a $\Sigma \Delta \mathrm{M}$ [17]-[19] that benefits from both the reduced error of a multi-bit quantizer and the inherent linearity of a single-bit quantizer in a single modulator. Single-loop EM- $\Sigma \Delta$ Ms that use this technique were reported in [20]-[22]. However, a MASH version of this technique has not been explored for use in electromechanical $\Sigma \Delta \mathrm{Ms}$ despite its potential advantages compared to the single loop approach. Such a version would rely on digitizing the signal of the forward path by a multi-bit ADC, which allows the signal to be processed in the digital domain. The structure uses the most-significant-bit (MSB) to close the first $\Sigma \Delta \mathrm{M}$ loop, while the remaining bits represent the quantization error of the single-bit quantizer, which can be then removed by a cancellation logical unit [17], [18]. This architecture can be referred to as MASHn-0 (where $\mathrm{n}$ is the $\Sigma \Delta \mathrm{M}$ order).

This paper presents for the first time the study of an electromechanical MASH- $\Sigma \Delta \mathrm{M}$ that employs the dual quantization technique and adopts an electromechanical 2-0 multi-stage noise shaping structure (EM-MASH2-0). The modulator consists of a second-order EM- $\Sigma \Delta \mathrm{M}$ accelerometer interfaced to a multi-bit quantizer, which is then followed by a digital compensator and a single-bit quantizer controlling an electrostatic feedback force to close the loop. Both quantization noise sources that are generated by the two quantizers are shaped by the sensing element loop filter. However, as the single-bit quantization noise is considerably greater than the multi-bit quantization noise, representing the dominant noise of the system (as will be discussed in the next section), and as it is in a digital form, it can be cancelled directly by a digital filter without the need for a second-stage $\Sigma \Delta \mathrm{M}$ (as in a MASH2-2 $\Sigma \Delta \mathrm{M}$ ). This reduces the complexity of the digital filter compared to the filters required for a MASH2-2, thus, the digital filter matching is easier to achieve.

The sensor system was implemented using surface mount printed circuit board (PCB) technology. Most of the system's components were implemented in the digital domain using a field programmable gate array (FPGA), which reduces electronic crosstalk noise and temperature effects, as well as providing extra flexibility in terms of modifying the modulator's parameters. This approach also provides excellent matching between the digital filter and the modulator's digital parameters. Nevertheless, as in MASH2-2, the MASH2-0 structure is sensitive to the parameter tolerances of the micromachined sensing element, which will be discussed in more detail later. The accelerometer used in the study is fabricated with a silicon-on-insulator (SOI) wafer with a device layer thickness of $50 \mu \mathrm{m}$, and uses a dicing-free and dry-release process (as described in [23]).

The paper is organized as follows: Section II discusses the system modeling and noise analysis. The simulation results are presented in section III. The mechanical design of the high-g capacitive MEMS accelerometer, the SOI fabrication and parameter characterization are discussed in section IV. The hardware implementation of the MASH2-0 $\Sigma \Delta \mathrm{M}$ is presented in section V. Section VI presents the experimental results of the MASH2-0 accelerometer. Section VII concludes the paper.

\section{System Modeling AND NoISE ANALysis}

The system-level view of the MASH2-0 control system is illustrated by the Simulink model in Fig. 1. The MASH2-0 consists of a micromachined capacitive MEMS accelerometer (M) embedded in a digitally controlled force-feedback loop that forms a second order electromechanical $\Sigma \Delta \mathrm{M}$. The differential sense capacitance 


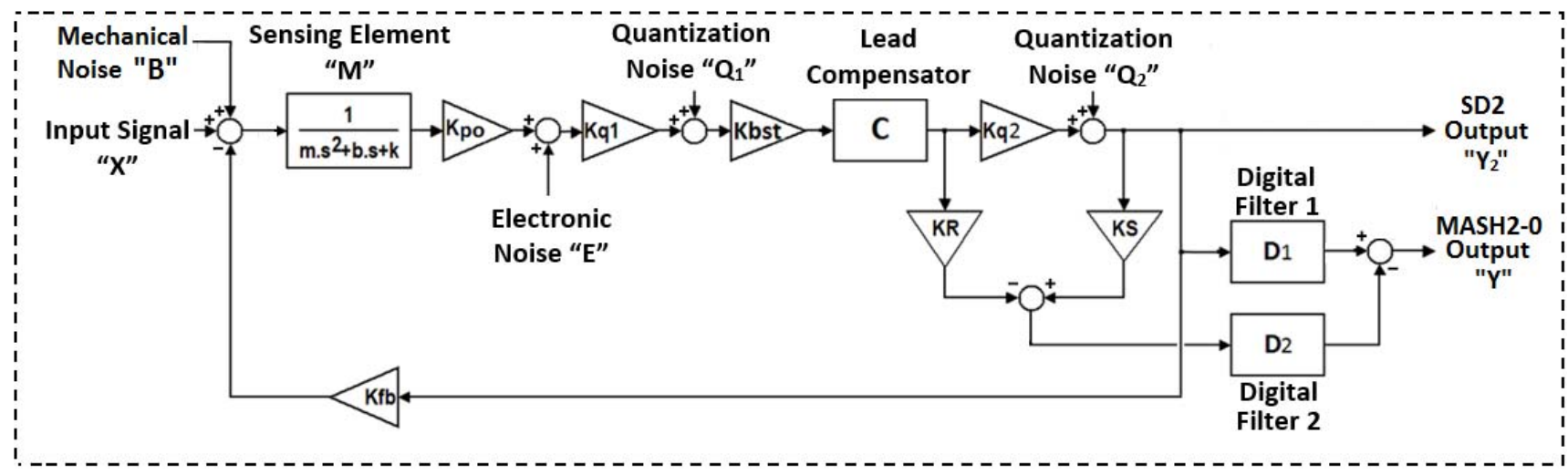

Fig. 2. Linear model of the MASH2-0 electromechanical $\Sigma \Delta \mathrm{M}$ shows the linearized quantization noises Q1 and Q2, and includes the mechanical noise of the MEMS accelerometer and electronic noise generated by the pickoff circuit.

change due to the acceleration input is converted by the pickoff circuit into a proportional voltage, which is then digitized by a multi-bit quantizer. A digital compensator (C) is used to maintain loop stability. This design retains the intrinsic linearity of single-bit feedback by using a single-bit quantizer to generate feedback pulses in order to maintain the proof mass at its nominal position. To achieve a performance higher than an electromechanical second-order $\Sigma \Delta \mathrm{M}$, the single-bit quantization noise must be cancelled by the digital filters.

The multi-bit quantization noise $(\mathrm{Q} 1)$ is considerably smaller than the single-bit quantization noise (Q2), making Q2 the dominant noise of the system. Because Q2 is in a digital form, it can be cancelled directly by digital filters without the need for a second-stage $\Sigma \Delta \mathrm{M}$, as necessary for a MASH2-2- $\Sigma \Delta$ M.

In order to design the digital filters (D1 and D2) and to inspect the effects of the different noise sources, a generic linear model for the system was constructed as shown in Fig. 2. Assuming the input signal sufficiently busy [24], the two quantizers were modeled as gain constants $(K q 1, K q 2)$ and additive white noise signals $(Q 1, Q 2)$. For small mass deflection, pickoff and feedback circuits can also be modeled as simple gain constants (Kpo, Kbst, and $K f b)$, in which Q2 is scaled by gain constants (KR, KS) and then cancelled by digital filters (D1 and D2).

The output signal Y2 of the electromechanical second-order $\Sigma \Delta \mathrm{M}$ (SD2) can be derived from the linear model using the superposition principle, taking the following form:

$$
\begin{aligned}
Y 2= & \operatorname{STF}_{S D 2}(X+B)+Q 1 N T F_{S D 2} Q 1 \\
& +Q 2 N T F_{S D 2} Q 2+E N T F_{S D 2} E
\end{aligned}
$$

The overall system performance is now determined by four noise sources: Mechanical noise force (B) due to the Brownian motion of the proof mass, electronic noise (E) due to the thermal and the flicker noise generated by the pickoff circuit, and the quantization noises Q1 and Q2.

If $X$ is the input force due to the acceleration, the signal transfer function $\left(S T F_{S D 2}\right)$, the multi-bit quantization noise transfer function $\left(Q 1 N T F_{S D 2}\right)$, the single-bit quantization noise transfer function $\left(Q 2 N T F_{S D 2}\right)$, and the pickoff circuit electronic noise transfer function $\left(E N T F_{S D 2}\right)$ can be derived as follows:

$$
\begin{aligned}
S \mathrm{TF}_{S D 2} & =\frac{Y_{2}}{X}=\frac{\mathrm{MK}_{\mathrm{po}} \mathrm{K}_{\mathrm{bst}} \mathrm{CK}_{\mathrm{q} 1} \mathrm{~K}_{\mathrm{q} 2}}{1+\mathrm{MK}_{\mathrm{po}} \mathrm{K}_{\mathrm{bst}} \mathrm{CK}_{\mathrm{fb}} \mathrm{K}_{\mathrm{q} 1} \mathrm{~K}_{\mathrm{q} 2}} \\
Q 1 N T F_{S D 2} & =\frac{Y_{2}}{Q_{1}}=\frac{\mathrm{K}_{\mathrm{bst}} \mathrm{CK}_{\mathrm{q} 2}}{1+\mathrm{MK}_{\mathrm{po}} \mathrm{K}_{\mathrm{bst}} \mathrm{CK}_{\mathrm{fb}} \mathrm{K}_{\mathrm{q} 1} \mathrm{~K}_{\mathrm{q} 2}} \\
Q 2 N T F_{S D 2} & =\frac{Y_{2}}{Q_{2}}=\frac{1}{1+\mathrm{MK}_{\mathrm{po}} \mathrm{K}_{\mathrm{bst}} \mathrm{CK}_{\mathrm{fb}} \mathrm{K}_{\mathrm{q} 1} \mathrm{~K}_{\mathrm{q} 2}} \\
\text { ENT F F }_{S D 2} & =\frac{Y_{2}}{E}=\frac{\mathrm{K}_{\mathrm{bst}} \mathrm{CK}_{\mathrm{q} 1} \mathrm{~K}_{\mathrm{q} 2}}{1+\mathrm{MK}_{\mathrm{po}} \mathrm{K}_{\mathrm{bst}} \mathrm{CK}_{\mathrm{fb}} \mathrm{K}_{\mathrm{q} 1} \mathrm{~K}_{\mathrm{q} 2}}
\end{aligned}
$$

To calculate the dominant quantization noise in the system, the spectral density of the modulation noise for the multi-bit quantizer $\mathrm{N}_{\mathrm{SD} 2}(\mathrm{f})$ can be found by multiplying the quantization noise spectral density Q1(f) with the modulus of $Q 1 N T F_{S D 2}$ as follows [24]:

$$
N 1_{S D 2}(f)=Q 1(f)\left|Q 1 N T F_{S D 2}\right|
$$

For a busy input signal, the quantization noise spectral density Q1(f) is of the following form [24]:

$$
Q 1(f)=Q 1_{r m s} \sqrt{2 T_{s}}
$$

where $T s$ is the sampling time, and $\mathrm{Q} 1_{\mathrm{rms}}$ is the RMS value of the quantization noise in the following form:

$$
Q 1_{r m s}=\frac{\Delta}{\sqrt{12}}
$$

where $\Delta$ is the level spacing of the quantizer. Assuming a uniform quantizer, the level spacing $\Delta$ is:

$$
\Delta=\frac{V_{f s}}{2^{m}-1}
$$

where $\mathrm{V}_{\mathrm{fs}}$ is the full scale input voltage, and $m$ is the number of quantizer bits.

By rearranging the above equations, the spectral density of the multi-bit modulation noise $\mathrm{N}_{\mathrm{SD} 2}(\mathrm{f})$ at the output $\mathrm{Y} 2$ takes the following form:

$$
N 1_{S D 2}(f)=\sqrt{\frac{T_{s}}{6}}\left(\frac{V_{f s}}{2^{m}-1}\right)\left|Q 1 N T F_{S D 2}\right|
$$

Similarly, the spectral density of the modulation noise for the single-bit quantizer N2(f) can be calculated as:

$$
N 2_{S D 2}(f)=\sqrt{\frac{T_{s}}{6}}\left(V_{f s}\right)\left|Q 2 N T F_{S D 2}\right|
$$




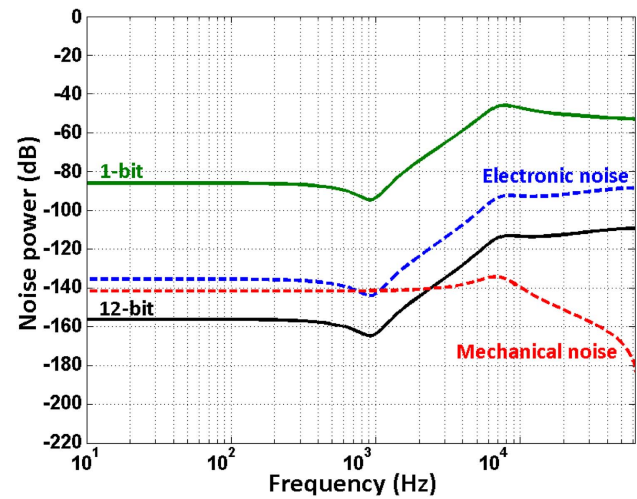

Fig. 3. Spectral density of the SD2 at output Y2 showing: the modulation noise of the 1-bit quantizer (green solid line), the 12-bit quantizer (black solid line), electronic noise (blue dashed line) and mechanical noise (red dashed line).

The electronic noise generated by the pickoff circuit is mainly thermal white noise. The flicker noise is largely suppressed as the pickoff circuit employs a high frequency carrier signal $(6 \mathrm{MHz})$ and a high pass filter, as will be discussed in section V-B. The equivalent electronic noise spectral density was estimated using the calculation presented in [25] and found to be $\mathrm{E}=15 \mu \mathrm{V} / \sqrt{ } \mathrm{Hz}$. Therefore, the electronic noise spectral density at the output of the modulator is given by:

$$
E_{S D 2}(f)=E\left|E N T F_{S D 2}\right|
$$

The mechanical noise spectral density is given by:

$$
B_{S D 2}(f)=B\left|S T F_{S D 2}\right|
$$

The mechanical noise force (B) is given by:

$$
B=\sqrt{4 K_{B} T b}
$$

where $K_{B}$ is the Boltzmann constant, $T$ is the Kelvin temperature, $b$ is the damping coefficient.

Using the above equations with the system parameters listed in Table II and assuming a quantizer full scale input voltage of $5 \mathrm{~V}$, and a 12-bit quantizer, the spectral density of the noise sources are shown in Fig. 3.

It can be seen that the quantization noise of Q2 in the digital domain is the performance limiter of the SD2. Thus, it will be the focus of the MASH2-0 design to cancel it using the digital filters D1 and D2.

The output signal $\mathrm{Y}$ of the MASH2-0 can be derived from the linear model by using the superposition principle as follows:

$$
\begin{aligned}
Y= & S \operatorname{TF}_{M A S H 2-0}(X+B)+Q 1 N T F_{M A S H 2-0} Q 1 \\
& +Q 2 N T F_{M A S H 2-0} Q 2+E N T F_{M A S H 2-0} E
\end{aligned}
$$

where $\mathrm{STF}_{\mathrm{MASH} 2-0}$, Q1NTF $\mathrm{MASH}_{2-0}$, Q2NTF $\mathrm{MASH}_{2-0}$, and $\mathrm{ETF}_{\mathrm{MASH} 2-0}$ are given by:

$$
\begin{aligned}
& S T F_{M A S H 2-0} \\
& \quad=\frac{Y}{X}=S T F_{S D 2}\left[D_{1}-D_{2}\left(K_{S}-\frac{K_{R}}{K_{q 2}}\right)\right] \\
& Q 1 N T F_{M A S H 2-0} \\
& \quad=\frac{Y}{Q 1}=Q 1 N T F_{S D 2}\left[D_{1}-D_{2}\left(K_{S}-\frac{K_{R}}{K_{q 2}}\right)\right]
\end{aligned}
$$

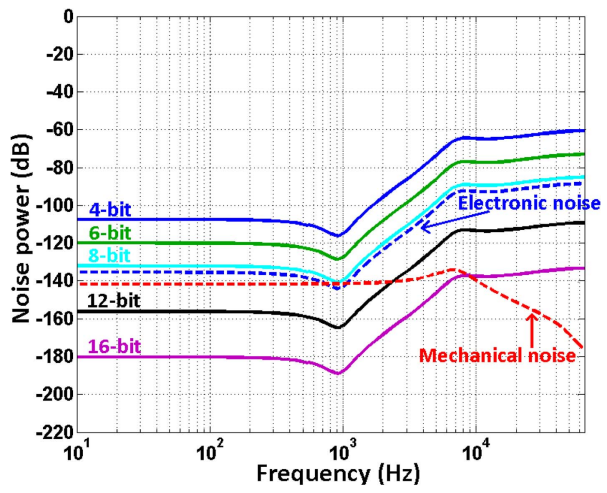

Fig. 4. Spectral density of the multi-bit modulation noise (ranging from 4 to 16-bit); the electronic and mechanical noises observed at the output Y.

$$
\begin{aligned}
& Q 2 N T F_{M A S H 2-0} \\
& =\frac{Y}{Q 2}=\left[\begin{array}{l}
D_{1} Q 2 N T F_{S D 2}-D_{2}\left(K_{S}-\frac{K_{R}}{K_{q 2}}\right) \\
\left.\quad \times Q 2 N T F_{S D 2}-D_{2}\left(\frac{K_{R}}{K_{q 2}}\right)\right]
\end{array}\right. \\
& \begin{aligned}
E N T F_{M A S H 2-0} \\
=\frac{Y}{E}=E N T F_{S D 2}\left[D_{1}-D_{2}\left(K_{S}-\frac{K_{R}}{K_{q 2}}\right)\right]
\end{aligned}
\end{aligned}
$$

In order to cancel the quantization noise of Q2, $Q 2 N T F_{M A S H 2-0}$ must equal zero. To achieve this, after some mathematical manipulation on (18), D2 takes the following form:

$$
D_{2}=\frac{D_{1} Q 2 N T F_{S D 2}}{\left(K_{S}-\frac{K_{R}}{K_{q 2}}\right) Q 2 N T F_{S D 2}+\left(\frac{K_{R}}{K_{q 2}}\right)}
$$

Note that if $\mathrm{D} 1, \mathrm{KS}, \mathrm{KR}$, and $\mathrm{Kq} 2$ equal one (i.e., they are unity gain constants), D2 and $\mathrm{Q}^{\mathrm{NTF}} \mathrm{MASH}_{\mathrm{MA}-0}$ can be simplified to:

$$
\begin{aligned}
D_{2} & =Q 2 N T F_{S D 2} \\
Q 1 N T F_{M A S H 2-0} & =Q 1 N T F_{S D 2}
\end{aligned}
$$

This simplifies the implementation of the digital filter D2.

If Q2 is completely cancelled, Q1, E and B noises will appear at the modulator output $\mathrm{Y}$. The spectral density of the modulation noise for the multi-bit quantizer $\mathrm{N}_{\mathrm{MASH}_{2}-0}(\mathrm{f})$ at the output of the MASH2-0 takes the following form:

$$
N 1_{\text {MASH2-O }}(f)=\sqrt{\frac{T_{s}}{6}}\left(\frac{V_{f s}}{2^{m}-1}\right)\left|Q 1 N T F_{M A S H 2-0}\right|
$$

Using the above equation and assuming a quantizer full scale input of $5 \mathrm{~V}$, the spectral densities of $\mathrm{N}_{\mathrm{MASH} 2-0}(\mathrm{f})$ for the multi-bit quantizeres (ranging from 4 to 16-bit) along with the electronic and mechanical noise are shown in Fig. 4. The figure shows that, the MASH2-0 performance can be evaluated as determined by the resolution of the multi-bit quantizer, which agrees with (17) and (22). It can also be observed that a high performance electromechanical $\Sigma \Delta \mathrm{M}$ can be realized with the dual quantization technique using a multi-bit quantizer of eight bits or more, such that the electronic and the mechanical noise are the performance limiter of the system. 


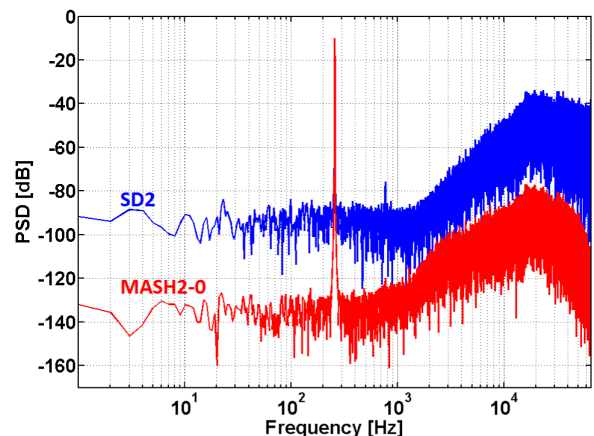

Fig. 5. Noise shaping of the SD2 (blue) and the MASH2-0 (red). The noise floor of the SD2 is about $-90 \mathrm{~dB}$, while the MASH2-0 is about $-130 \mathrm{~dB}$.

In this study, a 12-bit quantizer was chosen with a sampling rate of $131 \mathrm{kHz}$.

\section{System Modeling And Simulation Results}

\section{A. Noise Shaping}

The system was simulated using the Simulink model shown in Fig. 1 which included the nonlinear components of the system such as: the quantizers and the feedback switch circuit, along with the mentioned noise sources. The noise shaping of the SD2 at output Y2 is shown in Fig. 5 (blue line). The quantization noise Q2, which dominates over other noises in the system, is shaped by the electromechanical second order $\Sigma \Delta \mathrm{M}$ with a noise floor of around $-90 \mathrm{~dB}$ within a bandwidth of $1 \mathrm{kHz}$. With regard to the noise shaping of the MASH2-0 at the output Y (Fig. 5, red line), the noise floor is lowered by $40 \mathrm{~dB}$ and achieves $-130 \mathrm{~dB}$ within a bandwidth of $1 \mathrm{kHz}$. As expected, the digital filter cancelled out Q2. However, as shown in Fig. 4, the theoretical limit of the multibit quantization noise Q2 is $-155 \mathrm{~dB}$, which indicates that the electronic noise $\mathrm{E}$ is now the dominant noise of the MASH2-0.

\section{B. Overload Acceleration Input and Dynamic Range}

The overload acceleration input (OLA) is an important criterion in an electromechanical $\Sigma \Delta \mathrm{M}$; the closed loop sensor is only stable if operated within the OLA. Using the same sensing element parameters and a feedback voltage of $12 \mathrm{~V}$, a simulation analysis was carried out to compare the performance of the MASH2-0 with a 4th order single loop EM- $\Sigma \Delta \mathrm{M}$ (SD4) and an EM-MASH2-2 (described in [15]) in terms of the OLA, the signal to noise ratio (SNR) and the dynamic range. The result is shown in Fig. 6; it can be seen that MASH2-0 shares the advantage of MASH2-2 of having high OLA of $21 \mathrm{~g}$ and overload factor (OLF) of 0.85 compared with SD4 which only achieved an OLA of $12 \mathrm{~g}$ and an OLF of 0.4. However, MASH2-0 achieved higher SNR of $115 \mathrm{~dB}$ and higher dynamic range of $118 \mathrm{~dB}$ than MASH2-2 and SD4.

\section{System Linearity}

Since the proof mass deflection in the EM- $\Sigma \Delta \mathrm{M}$ typically is small, the nonlinear effects associated with the sense capacitors, squeeze film damping and the beam elasticity can be

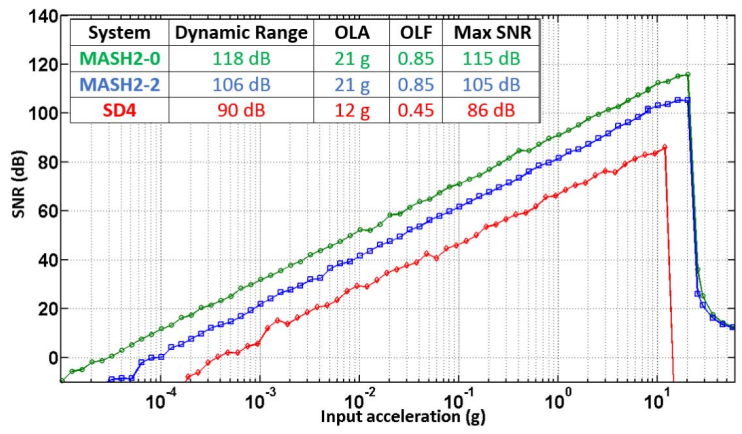

Fig. 6. Input acceleration (g) vs. SNR of the MASH2-0, MASH2-2 and SD4. MASH2-0 shows higher SNR and dynamic range compered with MASH22 and SD4.

considered as negligible [24]. Therefore, the nonlinearity of the MASH2-0 originates from the following main nonlinear sources:

First, the single-bit quantizer: The nonlinearity of the MASH2-0 is inherited from the first loop, which is an SD2. It is proven in the literature [26], [27] that the nonlinear behavior associated with the single-bit quantizer is more pronounced in low order $\Sigma \Delta \mathrm{M}$ than in higher order $(>2) \Sigma \Delta \mathrm{M}$. This is because, in the higher order $\Sigma \Delta \mathrm{M}$, the integrators in the forward path randomize the quantization error and make it independent of the input signal. To enhance the linearity of the SD2, and consequently of the MASH2-0, the dithering technique can be used [26], [27].

Second, the electrostatic force: During the closed loop operation, the applied electrostatic force $K_{f b}$ is given by:

$$
K_{f b}=\frac{\varepsilon_{o} * \text { Area }}{2}\left(\frac{V_{f b}}{d_{o}+x}\right)^{2}
$$

Although the EM- $\Sigma \Delta \mathrm{M}$ applies constant voltage pulses $V_{f b}$, the electrostatic force is nonlinear with regards to the residual proof mass movement $\mathrm{x}$; hence, the effective electrostatic force increases as the proof mass gets closer to the energized electrode. This has a nonlinear effect in the $\mathrm{EM}-\Sigma \Delta \mathrm{M}$, which produces odd $(3,5 \ldots)$ harmonic distortion signals with respect to the residual motion $\mathrm{x}$. A linearization technique proposed in [28] can be used to linearize the feedback force. Another solution is to design the sensor with a capacitive actuator with a lateral comb finger structure, which would produce better linear electrostatic force with respect to the proof mass movement [29].

The linearity of the MASH2-0 was investigated by simulation over an acceleration input range of $\pm 20 \mathrm{~g}$, as shown in Fig. 7. The maximum nonlinearity of the system was obtained in terms of a percentage of the maximum diversion of the simulated data from the straight line, which was found to be $1.14 \%$.

To study the effect of the above-mentioned nonlinear sources and to compare the linearity of the MASH2-0 with the SD4, the linearity test was repeated for both systems as follows:

1- Linearity test with dithering only.

2- Linearity test with feedback linearization only.

3- Linearity test with dithering and feedback linearization. 


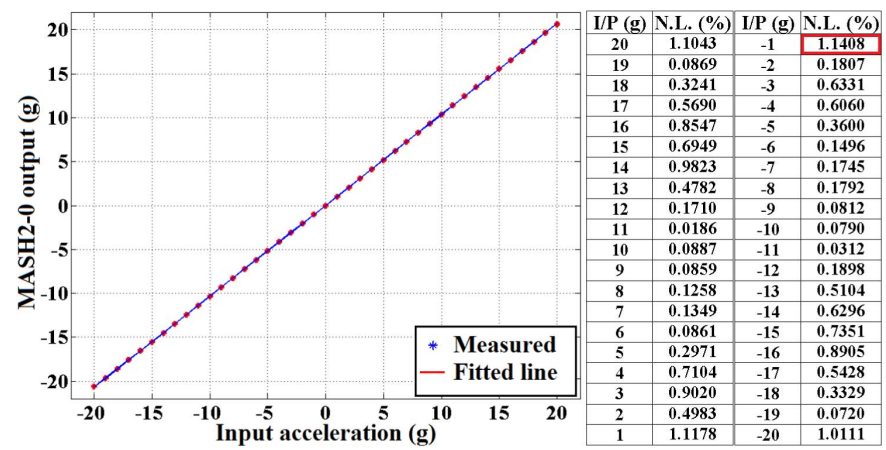

Fig. 7. Linearity response of the MASH2-0 for an input range of $\pm 20 \mathrm{~g}$.

TABLE I

MASH2-0 AND SD4 LINEARITY TEST

\begin{tabular}{ccccc}
\hline \hline & Test-1 & Test-1 & Test-3 & Test-4 \\
\hline MASH2-0 & $1.1408 \%$ & $0.8360 \%$ & $0.4022 \%$ & $0.3581 \%$ \\
SD4 & $0.8185 \%$ & $0.8242 \%$ & $0.4908 \%$ & $0.4978 \%$ \\
\hline \hline
\end{tabular}

The results are summarized in Table I. As can be seen for Test-1 for MASH2-0, the current system (No dithering, No feedback linearization) has a maximum nonlinearity of $1.14 \%$. However, in Test-2, the dithering improved the linearity response and achieved maximum nonlinearity of $0.83 \%$. In Test-3, the system exhibited a maximum nonlinearity of $0.4 \%$. It is clear that the feedback linearization in Test-3 considerably improved the linearity response, which indicates that the feedback linearization not only reduces the odd harmonic distortion signals but also improves the overall linearity of the system. In Test- 4 , with both dithering and feedback linearization included, the system achieved a maximum nonlinearity of $0.35 \%$.

The linearity of SD4 was investigated over an acceleration input range of $\pm 12 \mathrm{~g}$. Test- 1 for SD4 shows a maximum nonlinearity of $0.81 \%$. As expected, the addition of the dithering in Test-2 did not significantly improve the linearity, as the quantization error is randomized by the feedforward integrators [30]. Test-3, where the feedback linearization technique was implemented, showed noticeable improvements and achieved a maximum nonlinearity of $0.49 \%$. Finally, in Test -4 the system employs the dithering and the feedback linearization technique, achieving a maximum nonlinearity of $0.49 \%$.

In conclusion, without implementing the dithering and the feedback linearization methods, the SD4 showed higher linearity than MASH2-0 due to the existence of the integrators in the forward path that randomize and whiten the quantization error. However, by employing the dithering technique, the MASH2-0 achieved a linearity response comparable to that of the SD4. The linearity tests for the MASH2-0 and the SD4 showed that the systems nonlinearity mainly affected by the nonlinear behavior of the electrostatic force due to the residual proof mass movement.

\section{Parameter Sensitivity}

Further investigation was carried out to determine MASH2-0 performance in terms of sensing element parameter variation. Fig. 8 shows the performance of the MASH2-0 when

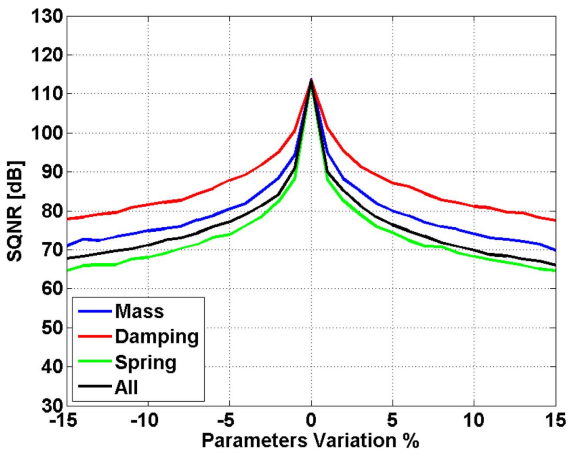

Fig. 8. MASH2-0 sensing element parameter sensitivity analysis, spring constant variation has the most effect on the MASH2-0 performance.

the parameters (proof mass (m), damping coefficient (b) and spring constant $(\mathrm{k})$ ) ranged between $\pm 15 \%$ of their nominal values. As expected, performance degraded accordingly, with the spring constant of the sensor having the most effect. However, the system attained robust stability performance despite the large variation.

Although, the MASH2-0 modulator offers a respectably high performance output, it is very sensitive to system parameter variations. The digital filter design process implies a comprehensive knowledge of the Q2NTF $\mathrm{SD}_{2}$ which is function of the sensing element parameters and other analogue parameters, both of which are subject to manufacturing tolerance and imperfections. This mismatch causes a leakage of the single-bit quantization noise to the output and degrades the performance of the MASH2-0. However, since MASH2-0 does not require a second stage $\Sigma \Delta \mathrm{M}$, the complexity of the digital filter $\mathrm{D} 2$ is reduced as indicated in (21). This will potentially allow the application of the adaptive control algorithms discussed in the electronic MASH $\Sigma \Delta \mathrm{M}$ [12], [31] to compensate for the filter mismatch. The implementation of the adaptive control for use with EM-MASH2-0 will be covered in another publication.

\section{Accelerometer Sensing Element Design}

\section{A. Accelerometer Theoretical Design}

The MEMS accelerometer used in this study is intended to measure acceleration input of $\pm 20 \mathrm{~g}$ when it is operated in closed loop $\Sigma \Delta \mathrm{M}$. Theoretically, small mass deflection can be achieved through closed loop operation so that the spring force can be neglected compared to the feedback force. Assuming small mass deflection, the feedback force $K_{f b}$ is given by the following formula [24]:

$$
K_{f b}=\frac{\varepsilon_{o} * \text { Area }}{2}\left(\frac{V_{f b}}{d_{o}}\right)^{2}
$$

where $\varepsilon_{o}$ is the air permittivity, Area is the total overlap area of the actuation capacitors, $V_{f b}$ is the applied feedback voltage, and $d_{o}$ is the nominal gap between the electrodes.

The maximum acceleration input $A c c_{\max }$ is estimated as follows:

$$
A c c_{\max }=\frac{K_{f b} * O L F}{\operatorname{mass} * 9.81}
$$




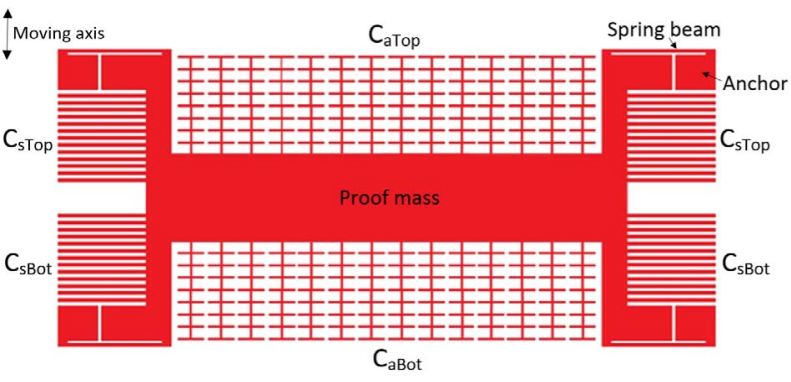

Fig. 9. Accelerometer layout structure; the proof mass is anchored to the substrate with four spring beams. The sense capacitors are on either sides of the sensor, and the actuator capacitive is suited in top and bottom of the sensor.

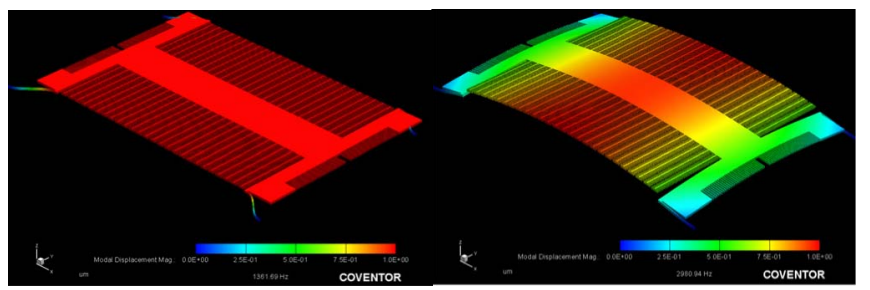

Fig. 10. FEM analysis (using CoventorWare) of the capacitive MEMS accelerometer. The first in-plane mode is at $1.361 \mathrm{kHz}$, and the out-of-plane mode is at $2.98 \mathrm{kHz}$.

By using the above equation and an OLF of 0.85 for the MASH2-0 as obtained in section III.B, a maximum input acceleration of $20 \mathrm{~g}$ acceleration is achievable by applying a feedback voltage of $9 \mathrm{~V}$.

The main motivation of the accelerometer design based on equations (25) and (26) was to maximize the feedback capacitor area and minimize the nominal gap. A simplified layout of the accelerometer is shown in Fig. 9. The sensing and feedback capacitors were designed to have a nominal gap of $4 \mu \mathrm{m}$. The set of capacitors $\left(C_{a T o p}\right.$ and $\left.C_{a B o t}\right)$ on the top and bottom serve as differential capacitive actuators, while the left and right $\left(C_{s T o p}\right.$ and $\left.C_{s B o t}\right)$ are differential sense capacitors. To accommodate as many actuators as possible, the proof mass is stretched horizontally, and the straight spring beams are located at the corners of the sensor. This enabled a maximum feedback area of around $7.5 \mu \mathrm{m}^{2}$.

The finite element method (FEM) analysis of the sensor is shown in Fig. 10. The first mode of the sensing element is an in-plane movement at a frequency of $1.36 \mathrm{kHz}$. In addition, the sensor was designed so that the out-of-plane movement is located at a higher frequency, roughly $2.9 \mathrm{kHz}$.

\section{B. Microfabrication}

A summary of the accelerometer fabrication steps is given in Fig. 11. The silicon-on-insulator (SOI) microfabrication process of the capacitive accelerometer was adopted from the dicing-free dry-release recipe described in [23]. The SOI wafer has a device layer of $50 \mu \mathrm{m}$, a buried oxide (BOX) layer of $2 \mu \mathrm{m}$, and a handle layer of $525 \mu \mathrm{m}$. The fabrication process was carried out using two masks: a device layer mask to define the sensor structure, and a handle layer mask to define the deep trenches, which were used to remove the handle layer
A)

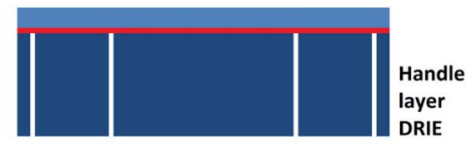

B)
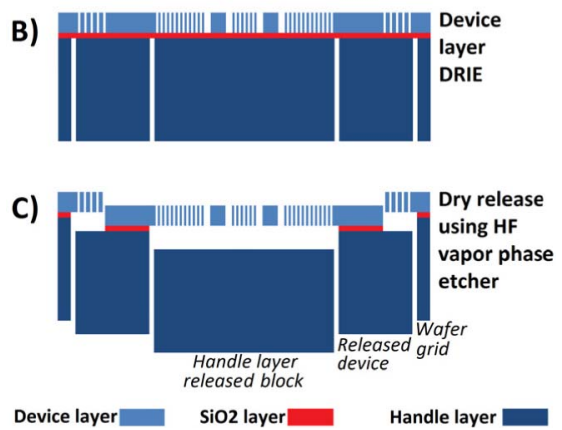

Fig. 11. SOI microfabrication steps of the high-g accelerometer sensor. A) is the handle layer DRIE step, B) is the device layer DRIE step, and C) is the dry release step using the $\mathrm{HF}$ vapour phase etcher.

block under the proof mass. The structure of the sensor was designed with a minimum feature size of $6 \mu \mathrm{m}$ and $4 \mu \mathrm{m}$ wide trenches.

The fabrication began (Fig. 11, step A) by defining the trench pattern on the handle layer, which involved a lithography procedure using photoresist AZ9260. The handle layer trenches were then processed using deep reactive ion etching (DRIE) down to the buried oxide layer. The photoresist mask on the handle layer was then stripped using photoresist solvent and treated with an oxygen plasma asher to guarantee that no photoresist remained. The second step of the fabrication (Fig. 11, step B) involved lithography of the device layer to pattern the wafer with the sensor's structural features. The process was carried out using AZ9260 photoresist, which was then followed by the DRIE process to etch the device layer down to the BOX.

The HF vapor phase etch technique was then used to etch the BOX layer (Fig. 11, step C). The release process was performed in three phases, beginning with the release of the proof mass, followed by the release of the handle layer block beneath the sensor, and finally, the release of the whole sensor off a remaining wafer grid. As the HF vapor phase etcher required a wafer to be processed top side facing down, the released handle layer block was supported by the sensor rather than the anchored proof mass, and the sensor block was supported by the wafer grid.

The top and bottom sides of the MEMS sensor are shown in the SEM images of Fig. 12. The design has four rest areas for the handle layer block so that, when fully released, the handle layer block is secured in terms of movement, and will not apply any pressure to the delicate anchored proof mass.

\section{Accelerometer Characterization}

The open loop frequency response of the accelerometer is shown in Fig. 13. The red line represents the acceleration input response of the sensor. The sensor was collocated to a reference sensor and excited using a shaker table. As can be seen, the resonance frequency is at $1.315 \mathrm{kHz}$, which closely 


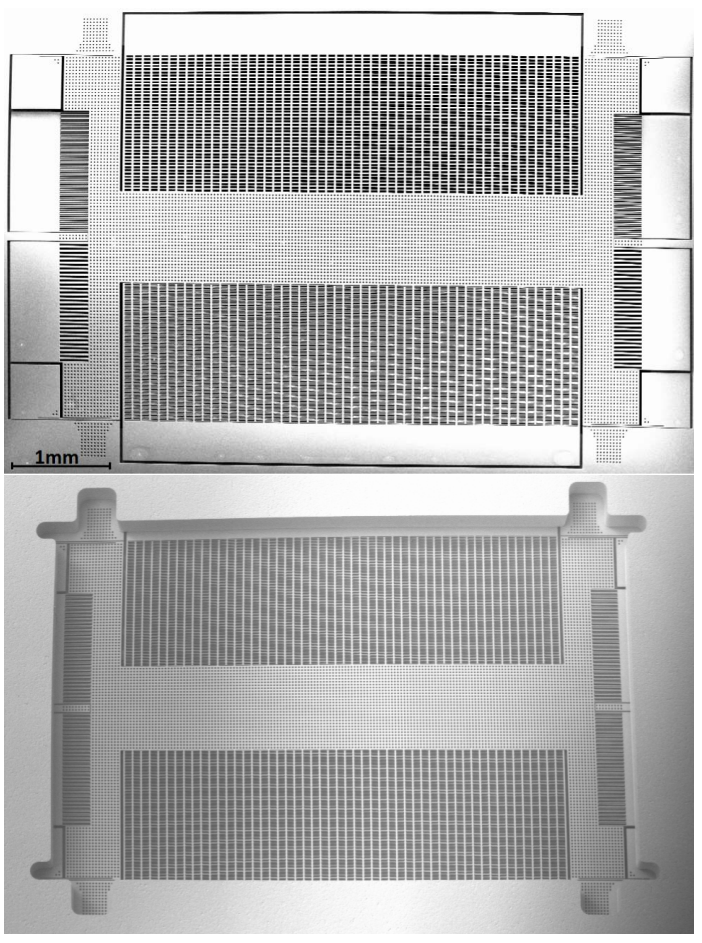

Fig. 12. SEM images of the fabricated accelerometer, (top) top view, and (bottom) bottom view of the sensor.

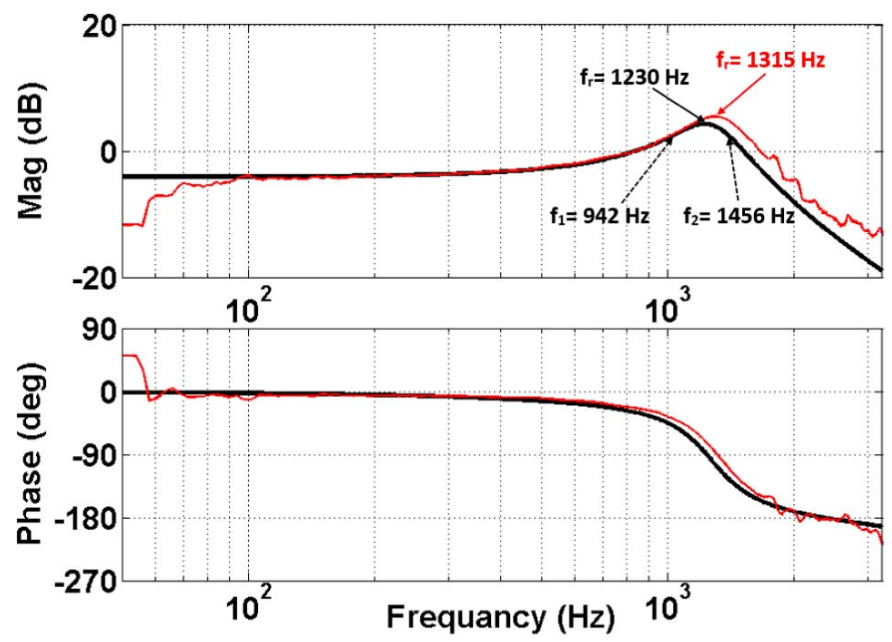

Fig. 13. Frequency response of the high-g accelerometer. The red line represents the acceleration input with a resonance frequency at $1315 \mathrm{~Hz}$, and the black line represents electrostatic force with a resonance frequency shifted down to $1230 \mathrm{~Hz}$ because of electrostatic spring softening, f1 $=942 \mathrm{~Hz}$ and $\mathrm{f} 2=1456 \mathrm{~Hz}$.

agrees with the FEM simulation. The black line represents the electrostatic force response, which was obtained by applying an excitation voltage on the top and bottom electrodes and observing the output. This type of measurement exhibits the spring softening phenomena [32], as a result, the resonance frequency shifts down to $1.23 \mathrm{kHz}$. Therefore, it will be considered when calculating the accelerometer parameters.

The design of the MASH2-0 accelerometer requires correct characterization of the sensing element. As discussed in section III.D, the digital filter D2 needs the lumped model
TABLE II

ACCELEROMETER AND MASh 2-0 System PARAMETERS

\begin{tabular}{clc}
\hline \hline Symbol & \multicolumn{1}{c}{ Description } & Value \\
\hline $\mathrm{m}$ & Proof mass $[\mathrm{kg}]$ & $1.59 \times 10^{-6}$ \\
$\mathrm{~b}$ & Damping coefficient $[\mathrm{N} . \mathrm{s} / \mathrm{m}]$ & $5.25 \times 10^{-3}$ \\
$\mathrm{k}$ & Spring constant $[\mathrm{N} / \mathrm{m}]$ & 99 \\
$\mathrm{~d}_{0}$ & Nominal gap between electrodes $[\mathrm{m}]$ & $4.5 \times 10^{-6}$ \\
$\mathrm{Cs}$ & Sense capacitor [F] & $2.5 \times 10^{-12}$ \\
$\mathrm{Cf}$ & Feedback capacitor $[\mathrm{F}]$ & $20.2 \times 10^{-12}$ \\
$\mathrm{Area}$ & Feedback capacitor overlap area $\left[\mathrm{m}^{2}\right]$ & $7.5 \times 10^{-6}$ \\
$\mathrm{Kpo}$ & Pickoff gain [V/m] & $5 \times 10^{6}$ \\
$\mathrm{Kbst}$ & Boost gain & 6 \\
$\mathrm{Zz}$ & Compensator zero $[\mathrm{Hz}]$ & $6 \times 10^{3}$ \\
$\mathrm{Pz}$ & Compensator pole $[\mathrm{Hz}]$ & $45 \times 10^{3}$ \\
$\mathrm{Fs}$ & Sampling frequency $[\mathrm{Hz}]$ & $131 \times 10^{3}$ \\
$\mathrm{Vfb}$ & Feedback voltage $[\mathrm{V}]$ & 12 \\
$\mathrm{KR}, \mathrm{KS}$ & Scaling factors & 1,1 \\
\hline \hline
\end{tabular}

parameters of the sensor; i.e. proof mass (m), damping coefficient (b) and spring constant (k).

The proof mass is derived from the fabrication mask layout, with the consideration of about 5\% loss due to the over-etching associated with the fabrication process. The proof mass $m$ was found to be:

$$
m=1.59 \times 10^{-6} \mathrm{~kg}
$$

The damping coefficient $b$ in capacitive MEMS accelerometer is mainly viscous air damping. Since the accelerometer is under-damped, the half-power method, as discussed in [33], is used to calculate the damping coefficient. The damping ratio $\zeta$ can be approximated by:

$$
\zeta=\frac{1}{2} \frac{f_{2}-f_{1}}{f_{r}}
$$

where $f_{r}$ is the resonance frequency and $f_{1}$ and $f_{2}$ are the half power frequency values as shown in Fig. 13. The estimated damping ratio $\zeta$ of the accelerometer based on the electrostatic force response is given by:

$$
\zeta=\frac{1}{2} \frac{1456-942}{1230}=0.2089
$$

If the $\omega_{n}$ is the natural frequency $(\mathrm{rad} / \mathrm{sec})$, the damping coefficient $\mathrm{b}$ can be calculated as follows:

$$
\begin{aligned}
\omega_{n} & =\frac{f_{r}}{\sqrt{1-\zeta^{2}}}=7.9027 \mathrm{k} \frac{\mathrm{rad}}{\mathrm{sec}} \\
b & =2 \zeta \omega_{n} m=5.25 \times 10^{-3} \frac{\mathrm{N.s}}{\mathrm{m}}
\end{aligned}
$$

As discussed in section 2, the spring constant is an important parameter that strongly changes the sensor dynamics during closed loop operation. It was calculated with the spring softening effect considered as follows:

$$
k=\omega_{n}^{2} \times m=99 \mathrm{~N} / \mathrm{m}
$$

The parameters of the accelerometer MEMS sensor and the electronic units are listed in Table II. 


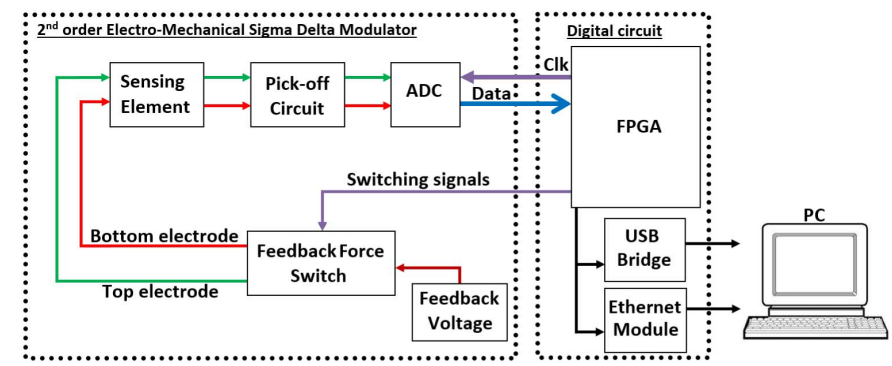

Fig. 14. MASH2-0 electronic circuit block diagram, showing the analogue and digital parts of the system.

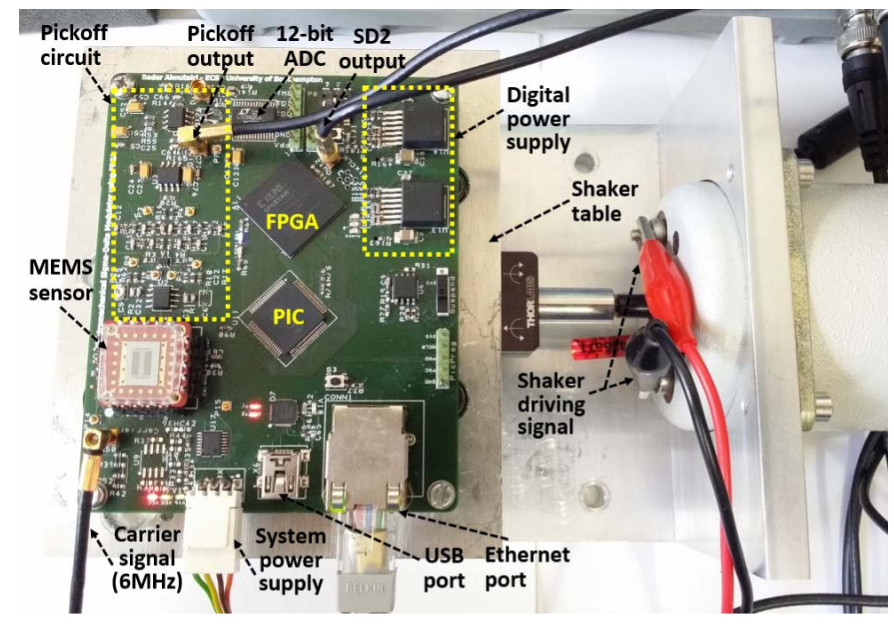

Fig. 15. MASH2-0 electronic circuit showing the different parts of the system along with the MEMS accelerometer sensor that was tested using a shaker table.

\section{HARDWARE IMPLEMENTATION}

\section{A. Electronic Circuit}

As shown in the block diagram in Fig. 14, the system is divided into analogue and digital circuit blocks. The second order electromechanical $\Sigma \Delta \mathrm{M}$ is fitted with analogue components that are designed with a fully differential signal path to eliminate common mode electronic noise signals within the analogue system. The analogue circuit accommodates the sensing element, which is followed by a pickoff circuit that measures the change in capacitance due to the acceleration and outputs a proportional voltage signal. The output signal of the pickoff is then converted into a digital signal using a 12-bit ADC. In order to close the loop, the capacitive actuators (top and bottom) are excited by the feedback pulse signals. The digital circuit consists of an FPGA unit. This unit reads the 12-bit data from the ADC at sampling rate of $131 \mathrm{kHz}$, performs the MASH2-0 digital filtering, and then outputs the feedback switching signals and MASH2-0 acceleration output. The system is designed to operate with USB and Ethernet ports to transmit real-time data. The MASH2-0 electronic circuit is shown in Fig. 15.

The pickoff circuit used in this study was constructed as shown in Fig. 16. The circuit comprises a simple differential capacitance to voltage converter (CVC) that utilizes an amplitude modulation (AM) technique [25], [34].

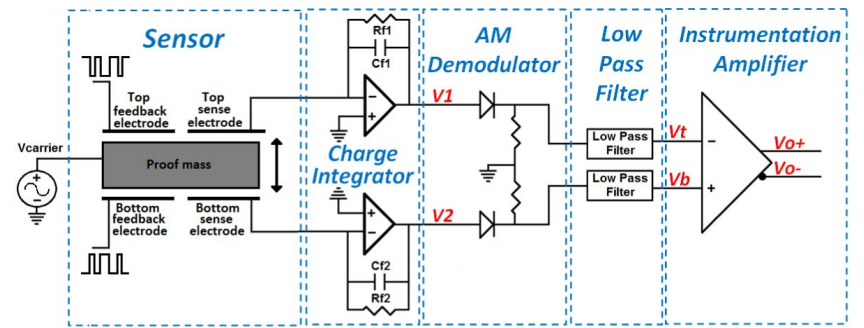

Fig. 16. Differential line pickoff circuit schematic diagram, which consists of a charge integrator, AM demodulators, and an instrumentation amplifier unit.

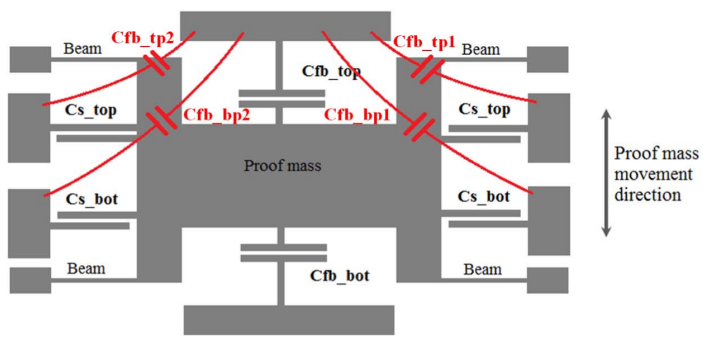

Fig. 17. Feedback parasitic capacitor model are connected to the sense capacitors, thus, the feedback pulses are observed on the pickoff output.

Both differential lines are symmetrical and constructed using: two half ac-bridges charge integrators, which act as AM modulators, two AM demodulators, and an instrumentation amplifier that rejects common mode signals.

Due to the proof mass displacement resulting from the acceleration, a differential change in sense capacitance alters the amplitude of the charge integrator output, hence, an amplitude modulation is achieved. The AM demodulator consists of a diode rectifier that rectifies the AM signal and leaves only its positive part to pass to the low pass filter. The low pass filter removes the high frequency component and recovers the low frequency acceleration signal. Finally, the instrumentation amplifier combines the differential movement of the proof mass and rejects the common mode signals.

\section{B. Effect of the Feedback Pulses Cross Talk}

One of the major problems in the design of closed loop accelerometers is the cross-talk between the feedback and sense capacitors. The feedback signal, which occurs in the form of pulses, is coupled into the sense capacitor. The coupling is due to the parasitic capacitance $\mathrm{C}_{\mathrm{fb}} \mathrm{p} 1,2$ and $\mathrm{C}_{\mathrm{fb}} \mathrm{p} 1,2$, as shown in Fig. 17. If the top feedback electrode is energized, and the bottom is grounded, the top parasitic capacitors $\mathrm{C}_{\mathrm{fb}} \mathrm{p} 1,2$ are closer to the energized electrode. Hence, a larger coupling signal is fed to the top sense capacitor, while the $\mathrm{C}_{\mathrm{fb}} \mathrm{p} 1,2$ is relatively far from the top electrode, and hence, a smaller coupling is fed to the bottom sense capacitor. Because of the difference in the coupled signal amplitude, the differential configuration of the pickoff circuit will not solve this issue.

During the digital closed loop operation, the feedback signal reaches a fundamental frequency equal to the limit cycle frequency, which was experimentally found to be $10 \mathrm{kHz}$. An experiment was carried out to study the effect of feedback 

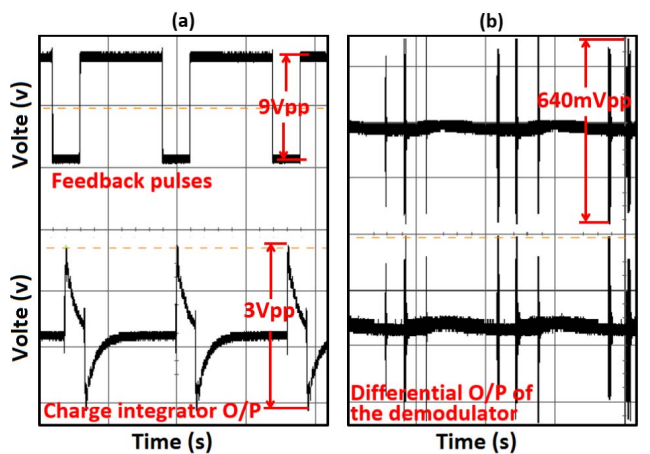

Fig. 18. (a) Upper trace is the feedback digital signal and lower trace is the output signal from charge integrator. (b) is the differential output of the pickoff circuit, which shows the feedback voltage cross coupling effect.

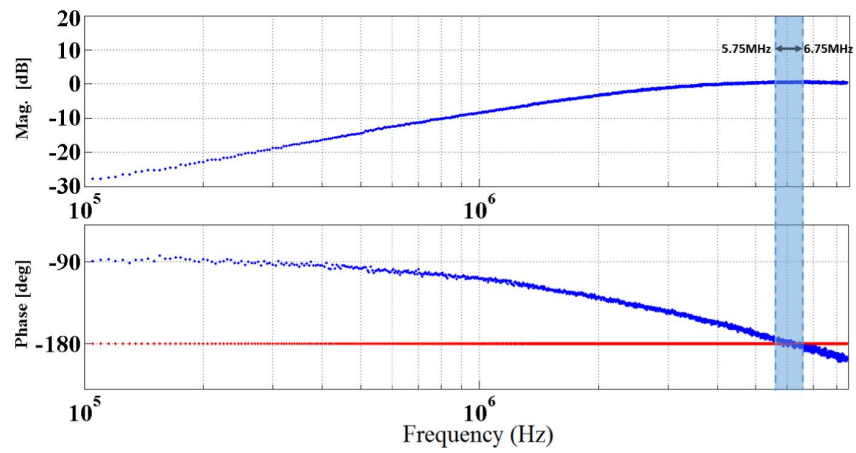

Fig. 19. Frequency response of the charge integrator. The carrier signal was chosen at $6 \mathrm{MHz}$, which is within the indicated region $(5.75-6.75 \mathrm{MHz}$ ).

parasitic capacitors. The proof mass was grounded, and both feedback electrodes were alternatively energized with pulses of 0 to $9 \mathrm{~V}$ amplitude and $10 \mathrm{kHz}$ frequency. Fig. 18-a shows the applied digital feedback signal in the upper trace, and the cross talk signal in the lower trace, observed at the output of the charge integrator. The output signal consisted of a low frequency component equal to the applied feedback signal frequency, and a high frequency component due to the rapid transition of the feedback signal; i.e., from $0 \rightarrow 9 \mathrm{~V}$. The differential output of the demodulator shown in Fig. 18-b is contaminated by the coupled signals; this strongly affects the performance of the closed loop sigma-delta modulator.

The cross talk signal can be eliminated by simply implementing a high pass or a band bass filter before the demodulator in the pickoff circuit. This will filter out the coupled signals caused by the digital feedback pulses. Therefore, the charge integrator was designed to offer high pass filtering with cut-off frequency of $3 \mathrm{MHz}$ by choosing a small feedback resistance $(\mathrm{RF}=10 \mathrm{k} \Omega)$ with the appropriate feedback capacitance. The Bode plot of the charge integrator is shown Fig. 19. The carrier signal is chosen in the narrow region between $5.73 \mathrm{M}$ to $6.73 \mathrm{MHz}$ where the response of the charge integrator gives $180^{\circ}$ phase shift. The low pass filtering during the demodulation process also ensures that any high frequency component is filtered out. Thus, only the modulated acceleration signal (i.e., proof mass movement), will pass through the high pass and low pass filters unaffected.

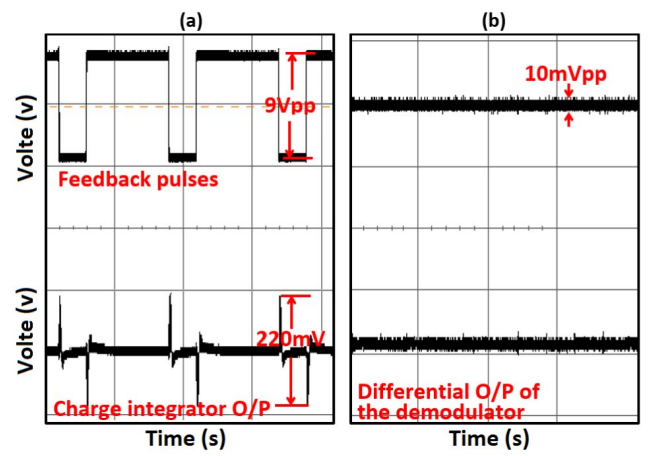

Fig. 20. (a) Upper trace is the feedback digital signal and lower trace is the output signal from charge integrator due to the coupling, but it is noticeably reduced due to the high pass effect. (b) is the differential output of the pickoff circuit, which shows no visible feedback voltage cross coupling effect after the low pass filter.

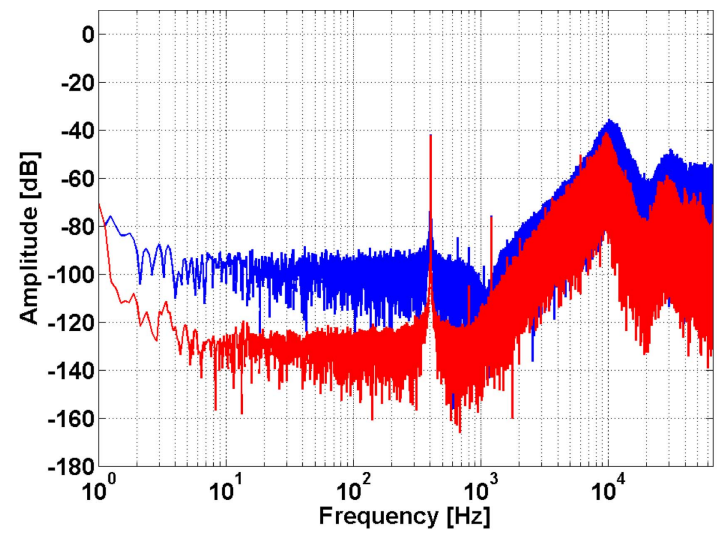

Fig. 21. Measured noise shaping of the SD2 (blue), with a noise floor of roughly $-90 \mathrm{~dB}$ above $10 \mathrm{~Hz}$, and the MASH2-0 (red), with a noise floor of roughly $-130 \mathrm{~dB}$ above $10 \mathrm{~Hz}$.

The results are shown in Fig. 20-a; the output of the charge integrator signal is the lower signal which shows that, only the high frequency component due to the rapid transition of the feedback signal is passed through the charge integrator. Fig. $20 \mathrm{~b}$ shows the differential output of the demodulator for both sense electrodes, where the coupled signals are heavily attenuated.

\section{EXPERIMENTAL RESULTS}

Dynamic measurements were taken for MASH2-0 and SD2 using a shaker table set to a sinusoidal acceleration of $0.5 \mathrm{~g}$ at $400 \mathrm{~Hz}$ as shown in Fig. 21. Above $10 \mathrm{~Hz}$, both results are in good agreement with the simulated output power spectra density shown in Fig. 5. The noise floor of the SD2 output (in blue) was roughly $-90 \mathrm{~dB}$, and the MASH2-0 output (in red) achieved a noise floor of approximately $-130 \mathrm{~dB}$. The MASH2-0 enhanced the performance of the system by roughly $40 \mathrm{~dB}$. Due to the fabrication tolerance, a mismatch between the top and bottom sense electrodes was introduced. This mismatch resulted in a DC component at the output of the MASH, hence the spectrum indicated a $-70 \mathrm{~dB}$ at DC, as shown in Fig. 21. Furthermore, any mismatch between the top and bottom differential lines of the pickoff circuit, will also 


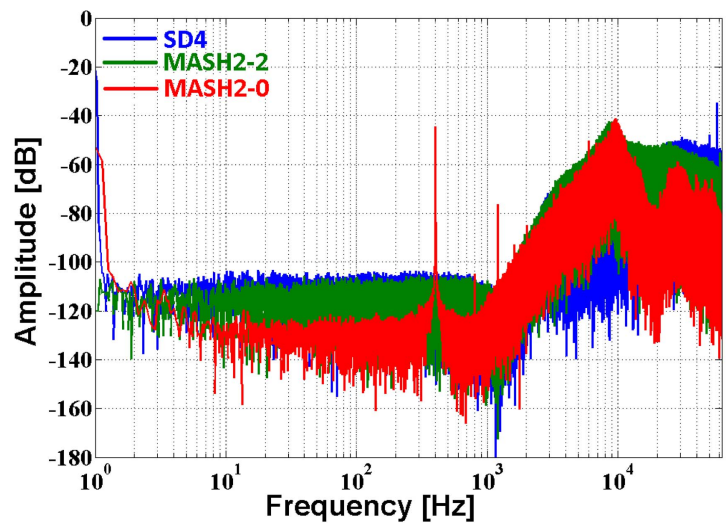

Fig. 22. Measured noise shaping of the SD4 (blue), with a noise floor of $-110 \mathrm{~dB}$, the MASH2-2 (green), with a noise floor of $-110 \mathrm{~dB}$, and the MASH2-0 (red), with a noise floor of $-130 \mathrm{~dB}$.

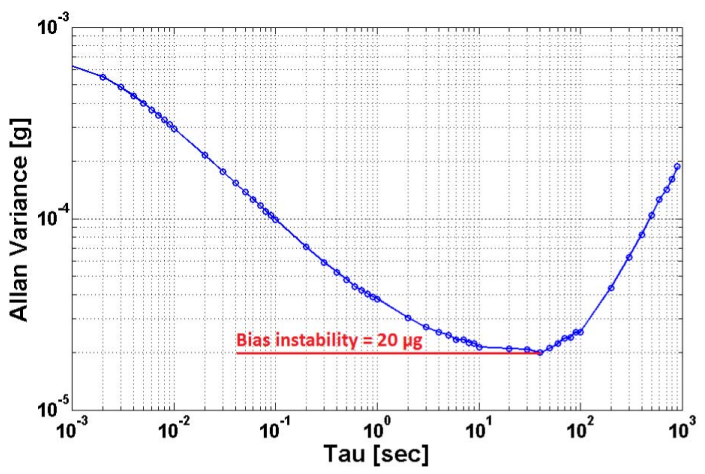

Fig. 23. Allan variance instability analysis of the MASH2-0 accelerometer shows a bias instability as low as $20 \mu \mathrm{g}$ at an integration time of 40 seconds.

result in a DC offset. One solution is to employ a DC nulling circuit after the pickoff circuit.

The MASH2-0 performance was experimentally compared with a fourth order single loop SD4 and a MASH2 2, which were both implemented on the same PCB board by programming the FPGA accordingly. The results are shown in Fig. 22. The SD4 (in blue) and MASH2-2 (in green) show fourth order noise shaping with a noise floor around $-110 \mathrm{~dB}$. Clearly, the MASH2-0 (in red) achieved a lower noise floor of $-130 \mathrm{~dB}$.

The output of the MASH2-0 accelerometer was observed for a period of three hours. The gathered data were processed to calculate bias instability using the Allan variance method [35]. The measurement showed a bias instability as low as $20 \mu \mathrm{g}$ at an integration time of 40 seconds, as shown in Fig. 23.

The MASH2-0 modulator was experimentally tested with another accelerometer from the same fabrication batch that had a parameter variation of roughly $10 \%$ compared to the original accelerometer. As expected, the modulator exhibited performance degradation, achieving a noise floor of roughly $-110 \mathrm{~dB}$, as shown in Fig. 24. However, it attained $20 \mathrm{~dB}$ lower noise floor compared with the SD2 shown in Fig. 21.

The linearity of the MASH2-0 was experimentally measured, as shown in Fig. 25. The system has a linear response over static acceleration range $\pm 1 \mathrm{~g}$. The maximum nonlinearity is calculated based on the worst diversion of

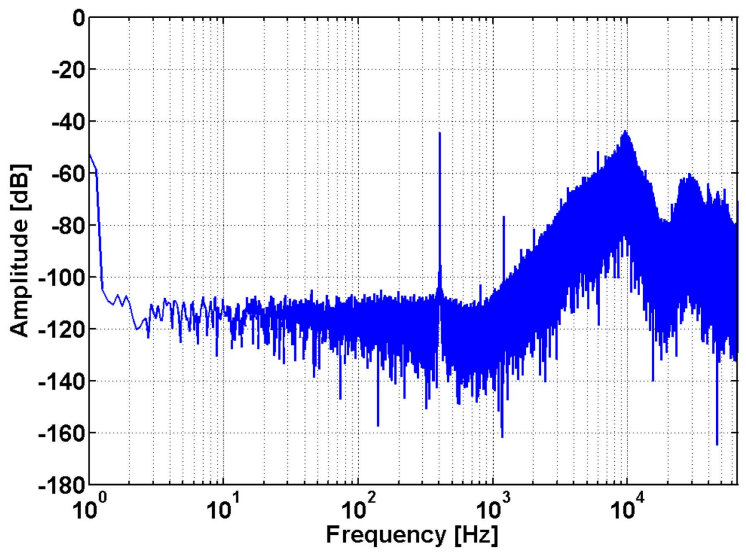

Fig. 24. Measured noise shaping of the MASH2-0 after the accelerometer was replaced with another of $10 \%$ parameter variation. The noise floor increased to roughly $-110 \mathrm{~dB}$.

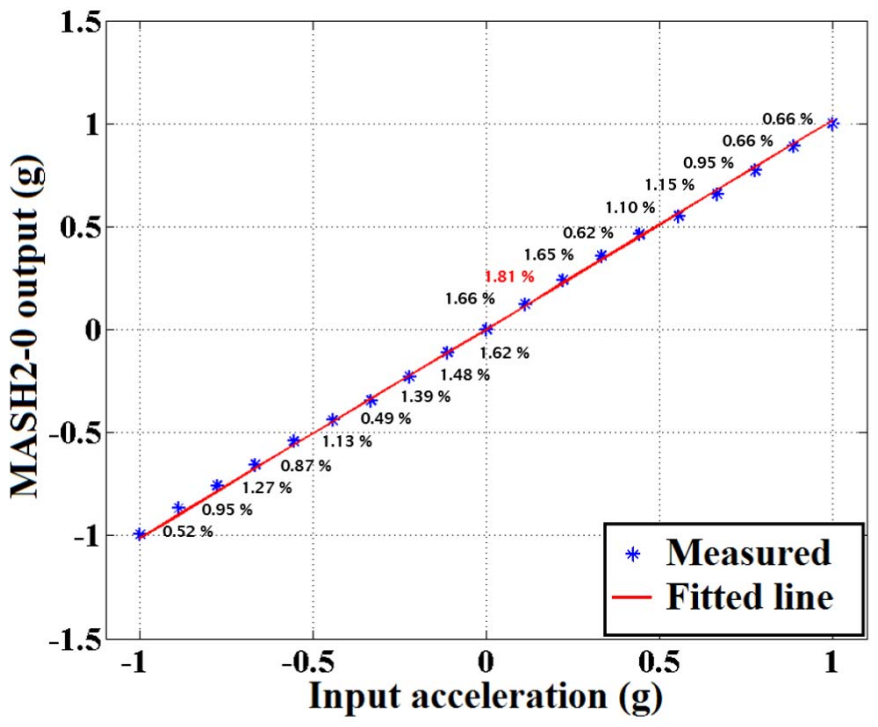

Fig. 25. Measured linearity response over static acceleration range $\pm 1 \mathrm{~g}$.

the measured result with the straight line, and is found to be $1.81 \%$. As discussed in section III.C the linearity of the system can be improved by employing dithering and feedback linearization methods.

\section{CONCLUSION}

This paper presented the theoretical and experimental analysis of a novel MASH2-0 EM- $\Sigma \Delta \mathrm{M}$ applied to an accelerometer implemented using the dual quantization technique. A capacitive MEMS accelerometer was designed on this study to achieve high input acceleration of $20 \mathrm{~g}$ when it is operated in digital closed loop operation. The simulation analysis of the MASH2-0 accelerometer showed that the performance of the second order EM- $\Sigma \Delta \mathrm{M}$ (SD2) is enhanced by around $40 \mathrm{~dB}$ in terms of the noise floor.

The experimental analysis was carried out with a sampling frequency of $131 \mathrm{kHz}$, the noise floor of the power spectral density of the SD2 output was around $-90 \mathrm{~dB}$, while the MASH2-0 showed a high performance output that reached 
around $-130 \mathrm{~dB}$ noise floor within a bandwidth of $1 \mathrm{kHz}$. The sensor achieved a maximum nonlinearity of $1.81 \%$ and a bias instability as low as $20 \mu \mathrm{g}$ at an integration time of 40 seconds.

The experimental measurements of the study closely matched the simulated measurements and prove that the MASH2-0 structure is applicable for high performance accelerometer.

The MASH2-0 accelerometer shares the same advantages of the MASH2-2 in term of robust stability, high overload input acceleration and linearity compared with single loop EM- $\Sigma \Delta$ M. Furthermore, MASH2-0 reduces the complexity of MASH2-2 and adds flexibility to design the digital filter. Nevertheless, as in MASH2-2, the MASH2-0 is sensitive to parameter variations which can potentially be addressed by an adaptive control algorithm.

\section{REFERENCES}

[1] W. Henrion, L. DiSanza, M. Ip, S. Terry, and H. Jerman, "Wide dynamic range direct accelerometer," in 4th Tech. Dig. Solid-State Sens. Actuator Workshop, Jun. 1990, pp. 153-157.

[2] Y. Dong, M. Kraft, C. Gollasch, and W. Redman-White, "A highperformance accelerometer with a fifth-order sigma-delta modulator," J. Micromech. Microeng., vol. 15, no. 7, pp. S22-S29, 2005.

[3] V. P. Petkov and B. E. Boser, "A fourth-order $\Sigma \Delta$ interface for micromachined inertial sensors," IEEE J. Solid-State Circuits, vol. 40, no. 8, pp. 1602-1609, Aug. 2005.

[4] U. Sonmez, H. Kulah, and T. Akin, "A fourth order unconstrained $\Sigma \Delta$ capacitive accelerometer," in Proc. 16th Int. Solid-State Sens., Actuators, Microsyst. Conf. (TRANSDUCERS), Jun. 2011, pp. 707-710.

[5] B. Almutairi and M. Kraft, "Comparative study of multi stage noise shaping and single loop sigma-delta modulators for MEMS accelerometers," in Proc. 24th Eurosensors Conf., 2010, pp. 512-515.

[6] G. I. Bourdopoulos, A. Pnevmatikakis, V. Anastassopoulos, and T. Deliyannis, Delta-Sigma Modulators: Modeling, Design, and Applications. London, U.K.: Imperial College Press, 2003.

[7] V. P. Petkov and B. E. Boser, "High-order electromechanical $\Sigma \Delta$ modulation in micromachined inertial sensors," IEEE Trans. Circuits Syst. I, Reg. Papers, vol. 53, no. 5, pp. 1016-1022, May 2006.

[8] T. H. Pearce, "Cascading bandpass sigma-delta A-D converters," presented at the 2nd International Conference on Advanced A-D and D-A Conversion Techniques, and Their Applications, vol. 393. 1994, pp. 1-6.

[9] T. L. Brooks, D. H. Robertson, D. F. Kelly, A. Del Muro, and S. W. Harston, "A cascaded sigma-delta pipeline A/D converter with $1.25 \mathrm{MHz}$ signal bandwidth and $89 \mathrm{~dB}$ SNR," IEEE J. Solid-State Circuits, vol. 32, no. 12, pp. 1896-1906, Dec. 1997.

[10] X. Wang, U. Moon, M. Liu, and G. C. Temes, "Digital correlation technique for the estimation and correction of DAC errors in multibit MASH $\triangle \Sigma$ ADCs," presented at the IEEE International Symposium on Circuits and Systems (ISCAS), vol. 4. May 2002, pp. IV-691-IV-694.

[11] G. Cauwenberghs and G. C. Temes, "Adaptive digital correction of analog errors in MASH ADCs. I. Off-line and blind on-line calibration," IEEE Trans. Circuits Syst. II, Analog Digit. Signal Process., vol. 47, no. 7, pp. 621-628, Jul. 2000.

[12] P. Kiss et al., "Adaptive digital correction of analog errors in MASH ADCs. II. Correction using test-signal injection," IEEE Trans. Circuits Syst. II, Analog Digit. Signal Process., vol. 47, no. 7, pp. 629-638, Jul. 2000.

[13] N. Maghari, S. Kwon, G. C. Temes, and U. Moon, "Mixed-order sturdy MASH $\Delta-\Sigma$ modulator," in Proc. IEEE Int. Symp. Circuits Syst. (ISCAS), May 2007, pp. 257-260.

[14] N. Maghari and U.-K. Moon, "Multi-loop efficient sturdy MASH deltasigma modulators," in Proc. IEEE Int. Symp. Circuits Syst. (ISCAS), May 2008, pp. 1216-1219.

[15] B. Almutairi and M. Kraft, "Experimental study of single loop sigmadelta and multi stage noise shaping (MASH) modulators for MEMS accelerometer,' in Proc. IEEE Sensors, Oct. 2011, pp. 520-523.

[16] B. Almutairi and M. Kraft, "Multi stage noise shaping sigma-delta modulator (MASH) for capacitive MEMS accelerometers," Sens. Actuators A, Phys., vol. 186, pp. 169-177, Oct. 2012.
[17] T. C. Leslie and B. Singh, "An improved sigma-delta modulator architecture," in Proc. IEEE Int. Symp. Circuits Syst., vol. 1. May 1990, pp. 372-375.

[18] N. Sakimura, M. Yamaguchi, and M. Yotsuyanagi, "Multibit delta-sigma architectures with two-level feedback loop using a dual-quantization architecture," IEICE Trans. Fundam. Electron., Commun. Comput. Sci. vol. E84-A, no. 2, pp. 497-505, 2001.

[19] R. F. Rio, F. M. Hidalgo, B. Perez-Verdue, J. M. de la Rosa, and A. Rodriguez-Vazquez, CMOS Cascade Sigma-Delta Modulators for Sensors and Telecom: Error Analysis and Practical Design. Berlin, Germany: Springer-Verlag, 2006.

[20] M. Pastre et al., "A $300 \mathrm{~Hz} 19$ b DR capacitive accelerometer based on a versatile front end in a $5^{\text {th }}$-order $\Delta \Sigma$ loop," in Proc. ESSCIRC, Sep. 2009, pp. 288-291.

[21] P. Zwahlen, A.-M. Nguyen, Y. Dong, F. Rudolf, M. Pastre, and H. Schmid, "Navigation grade MEMS accelerometer," in Proc. 23rd IEEE MEMS, Hong Kong, Jan. 2010, pp. 631-634.

[22] Y. Dong, P. Zwahlen, A. M. Nguyen, R. Frosio, and F. Rudolf, "Ultra-high precision MEMS accelerometer," in Proc. 16th Int. Solid-State Sens., Actuators, Microsyst. Conf. (TRANSDUCERS), Jun. 2011, pp. 695-698.

[23] I. Sari, I. Zeimpekis, and M. Kraft, "A dicing free SOI process for MEMS devices," Microelectron. Eng., vol. 95, pp. 121-129, Jul. 2012.

[24] V. Kaajakari, Practical MEMS. Las Vegas, NV, USA: Small Gear, 2009.

[25] J. C. Lotters, W. Olthuis, P. H. Veltink, and P. Bergveld, "A sensitive differential capacitance to voltage converter for sensor applications," IEEE Trans. Instrum. Meas., vol. 48, no. 1, pp. 89-96, Feb. 1999.

[26] E. Janssen and A. van Roermund, Look-Ahead Based Sigma-Delta Modulation. New York, NY, USA: Springer, 2011.

[27] S. E. Norsworthy, R. Schreier, and G. C. Temes, Delta-Sigma Data Converters: Theory, Design, and Simulation. Hoboken, NJ, USA: Wiley, 1997.

[28] Y. Dong, M. Kraft, and W. Redman-White, "Force feedback linearization for higher-order electromechanical sigma-delta modulators," J. Micromech. Microeng., vol. 16, no. 6, pp. S54-S60, 2006.

[29] C. Acar and A. Shkel, MEMS Vibratory Gyroscopes: Structural Approaches to Improve Robustness. New York, NY, USA: Springer, 2009.

[30] J. M. de la Rosa, "Sigma-delta modulators: Tutorial overview, design guide, and state-of-the-art survey," IEEE Trans. Circuits Syst. I, Reg. Papers, vol. 58, no. 1, pp. 1-21, Jan. 2011.

[31] S.-C. Lee and Y. Chiu, "A 15-MHz bandwidth 1-0 MASH $\Sigma \Delta$ ADC with nonlinear memory error calibration achieving 85-dBc SFDR," IEEE J. Solid-State Circuits, vol. 49, no. 3, pp. 695-707, Mar. 2014.

[32] V. Kempe, Inertial MEMS: Principles and Practice. Cambridge, U.K.: Cambridge Univ. Press, 2011.

[33] L. Meirovitch, Fundamentals of Vibrations. New York, NY, USA: McGraw-Hill, 2001.

[34] S. D. Senturia, Microsystem Design. New York, NY, USA: Kluwer, 2002.

[35] N. El-Sheimy, H. Hou, and X. Niu, "Analysis and modeling of inertial sensors using Allan variance," IEEE Trans. Instrum. Meas., vol. 57, no. 1 , pp. 140-149, Jan. 2008

Bader Almutairi received the B.Sc. degree in computer engineering from King Saud University, Riyadh, Saudi Arabia, in 2003; the M.Sc. (Hons.) degree in embedded digital systems from the University of Sussex, Brighton, U.K., in 2008; and the Ph.D. degree from the University of Southampton, in 2015. He joined the Space Research Institute, King Abdulaziz City for Science and Technology, (KACST), Riyadh, in 2003, where he worked on control and navigation systems design. In 2009, he joined the Nano Research Group, University of Southampton, to study in the field of inertial microelectromechanical systems (MEMS) sensors and advanced control systems for MEMS devices. He is currently with KACST, where he is applying his expertise in MEMS and advanced control systems. 
Ali Alshehri was born in Riyadh, Saudi Arabia, in 1980. He received the B.Sc. degree in computer engineering from King Saud University, Riyadh, in 2003; the M.Sc. (Hons.) degree in embedded digital systems from the University of Sussex, Brighton, U.K., in 2008; and the Ph.D. degree in electronic and electrical engineering with a focus on the field of inertial microelectromechanical systems (MEMS) sensors and advanced control systems for MEMS devices. Since 2003, he has been a Researcher with the Space Research Institute, King Abdulaziz City for Science and Technology, Riyadh, where he was involved in the design and implementation of control and navigation systems.
Michael Kraft was born in Nürnberg, Germany, in 1966. He received the Dipl.-Ing. (Univ.) degree in electrical and electronics engineering from the Friedrich Alexander Universität Erlangen-Nürnberg, in 1993, and the Ph.D. degree from Coventry University, U.K., in 1997. He joined the Nonlinear System Design Group, Coventry University, in 1993, where he worked on the design of a digital micromachined accelerometer. He then spent two years with the Berkeley Sensors and Actuator Center, University of California at Berkeley, USA, where he was working on the design of integrated micromachined gyroscopes. From 1999 to 2012, he was a Faculty Member and Professor of Microsystem Technology with the University of Southampton, U.K. He also served as the Director of the Southampton Nanofabrication Centre from 2010 to 2012. From 2012 to 2014, he was with the Fraunhofer Institute for Microelectronic Circuits and Systems, Duisburg, Germany, heading the Department of Micro- and Nanosystems, with a focus on fully integrated microsensors and biohybrid systems. Concurrently, he held the Professorial Chair (W3) of Integrated Micro- and Nanosystems with the University of Duisburg-Essen. In 2015, he joined the University of Liege, Belgium, as a Professor of Micro- and Nanosystems. His principle interests include microelectromechanical systems (MEMS) sensors and actuators, micro- and nanofabrication, intelligent control systems for MEMS devices, engineering of atom chips, and electronic circuit design. 